\title{
Implementación de la Técnica de los K-Vecinos en un Algoritmo de Recomendación para un Sistema de Compras Utilizando NFC y Android
}

\section{Implementation of the $\mathrm{k}$ - Neighbors Technique in a Recommender Algorithm for a Purchasing System Using NFC and Android}

\author{
DOI: http://dx.doi.org/10.17981/ingecuc.13.1.2017.01
}

Artículo de investigación. Fecha de recepción: 22 de agosto de 2016. Fecha de aceptación: 1 de diciembre de 2016

\author{
Oscar Arley Riveros Rey \\ Universidad Distrital Francisco José de Caldas. Bogotá (Colombia) \\ scrriveros252@gmail.com \\ Juan Guillermo Romero Fajardo \\ Universidad Distrital Francisco José de Caldas. Bogotá (Colombia) \\ jguillermo99@hotmail.com \\ Jhon Francined Herrera Cubides \\ Universidad Distrital Francisco José de Caldas. Bogotá (Colombia) \\ jfherrerac@udistrital.edu.co
}

Cómo citar este artículo:

O. Riveros Rey, J. Romero Fajardo y J. Herrera Cubides, "Implementación de la Técnica de los K-Vecinos en un Algoritmo de Recomendación para un Sistema de Compras Utilizando NFC y Android", INGE CUC, vol. 13, No.1, pp. 9-18, 2017. DOI: http://dx.doi. org/10.17981/ingecuc.13.1.2017.01

\section{Resumen}

Introducción- Este artículo se orienta en presentar el diseño de una aplicación móvil que involucra la tecnología NFC y un algoritmo recomendador colaborativo bajo la técnica de los K-vecinos, permitiendo observar sugerencias personalizadas para cada cliente.

Objetivo- Diseñar y desarrollar una aplicación móvil, usando tecnologías NFC y Técnica de los K-Vecinos en una Algoritmo recomendador, para un Sistema de Compras.

Metodología- El proceso seguido para el diseño y desarrollo de la aplicación se enfoca en:

- Revisión del estado del arte en los sistemas de compras móviles.

- Construcción del Estado del arte en el uso de la Tecnología NFC y las técnicas de IA para sistemas recomendadores, enfocadas en los Algoritmos de K-Vecinos

- Diseño del sistema Propuesto

- Parametrización e implementación de la Técnica de los K-Vecinos e integración de la Tecnología NFC

- Implementación y Pruebas del Sistema Propuesto.

Resultados- Dentro de los resultados obtenidos se detallan:

- Aplicación móvil que integra Android, Tecnologías NFC y una Técnica de Algoritmo Recomendador

- Parametrización de la Técnica de los K-Vecinos, para ser usada dentro del algoritmo recomendador.

- Implementación de requerimientos funcionales que permiten generar recomendaciones personalizadas de compra al usuario, calificaciones de usuario

Conclusiones- La técnica de los k-vecinos en un algoritmo recomendador permite suministrarle al cliente una serie recomendaciones con un nivel de seguridad, dado que este algoritmo realiza cálculos teniendo en cuenta múltiples parámetros y contrasta los resultados obtenidos para otros usuarios logrando encontrar los artículos con un mayor grado de similitud con el perfil del cliente. Este algoritmo parte de una muestra de productos similares, complementarios y otros no relacionados, aplicando su formulación respectiva, se obtiene que la recomendación se realiza únicamente con los productos complementarios que obtuvieron mayor calificación: marcando una gran diferencia con la mayoría de sistemas recomendadores del mercado, que sólo se limitan a sugerir los productos más vendidos, mejor calificados o de la misma categoría.

Palabras clave- Algoritmo recomendador, Android, aplicación, base de datos, móvil, productos, recomendador, retroalimentación, servidor.

\section{Abstract}

Introduction- This paper aims to present the design of a mobile application involving NFC technology and a collaborative recommendation algorithm under the K-neighbors technique, allowing to observe personalized suggestions for each client.

Objective- Design and develop a mobile application, using NFC technologies and K-Neighbors Technique in a recommendation algorithm, for a Procurement System.

Methodology- The process followed for the design and development of the application focuses on:

- Review of the state of the art in mobile shopping systems.

- State-of-the-art construction in the use of NFC technology and AI techniques for recommending systems, focused on K-Neighbors Algorithms

- Proposed system design

- Parameterization and implementation of the K-Neighbors Technique and integration of NFC Technology

- Proposed System Implementation and Testing

Results-Among the results obtained are detailed:

- Mobile application that integrates Android, NFC Technologies and a Technique of Algorithm Recommendation

Parameterization of the K-Neighbors Technique, to be used within the recommend algorithm.

- Implementation of functional requirements that allow the generation of personalized recommendations for purchase to the user, user ratings

Conclusions- The k-neighbors technique in a recommendation algorithm allows the client to provide a series of recommendations with a level of security, since this algorithm performs calculations taking into account multiple parameters and contrasts the results obtained for other users, finding the articles with a Greater degree of similarity with the customer profile. This algorithm starts from a sample of similar, complementary and other unrelated products, applying its respective formulation, we obtain that the recommendation is made only with the complementary products that obtained higher qualification; Making a big difference with most recommending systems on the market, which are limited to suggest the best-selling, bestqualified or in the same category.

Keywords- Algorithm, android, application, database, feedback, mobile, products, recommender, server. 


\section{Introducción}

La incursión de la tecnología en diferentes escenarios del diario vivir ha venido transformando la manera de ver, de pensar y también de comprar por parte de los individuos. Tal es el caso que en la actualidad, las grandes industrias han venido transformando sus procesos, optando por incorporar nuevas tecnologías que mejoren la experiencia del usuario. Un claro ejemplo se encuentra en la industria de bienes de consumo, que evoluciona de acuerdo con las tendencias del mercado y donde el cliente está en constante búsqueda de los beneficios digitales, tanto en las tiendas de abarrotes como de bienes perdurables [1].

Ahora mismo, pequeñas y grandes empresas han optado por incluir, dentro de sus portafolios de servicios, una gran variedad de avances tecnológicos con el objetivo de mejorar su utilidad, y lo más importante, fidelizar al cliente con experiencias personalizadas [2]. Para Netflix, una empresa comercial estadounidense, el cliente pierde el interés en la búsqueda de contenido digital (películas), pasados los 60 a 90 segundos de búsqueda, lo que trae consigo el abandono del servicio $\mathrm{y}$, por lo tanto, la pérdida del cliente [3].

Una solución que ha venido implementando Netflix es incorporar un sistema de recomendación, el cual se define como una serie de algoritmos encargados de recolectar información, ordenarla y clasificarla con la intención de presentar sugerencias personalizadas a cada uno de los miembros de la plataforma. El objetivo de este tipo de empresas, donde existe una gran variedad de contenido, es la implementación de sistemas de recomendación con la intención de conocer al usuario y ofrecer contenido que se encuentra caracterizado por los intereses del beneficiario.

El cliente actual y el del futuro están en constante búsqueda de nuevas experiencias que le permitan tomar las mejores decisiones. Es así que parten de la necesidad de automatizar y de generar mayores facilidades en las actividades diarias. De esta manera, las diferentes compañías se encuentran en constante búsqueda de las mejores experiencias de usuario [4]. Una tecnología que actualmente está generando nuevas formas de realizar las actividades cotidianas, tales como: la forma de pago, transferencia de datos y hasta de acceso a los hogares, es conocida como NFC [5].

NFC (Near Field Communication) es una tecnología emergente que permite a diferentes dispositivos comunicarse con otros a una distancia máxima de alrededor de $20 \mathrm{~cm}$, facilitando tareas, ya sea a nivel empresarial o personal [6].

Teniendo en cuenta lo anterior, el presente documento se centra en una aplicación móvil que involucra la tecnología NFC y un algoritmo de recomendación colaborativo como herramientas com- plementarias y de gran ayuda, tanto para clientes como para empresas, permitiendo una colaboración de parte y parte. La aplicación consiste en un sistema de adquisición de productos mediante dispositivos móviles que incorpora un algoritmo de recomendación bajo la técnica de los K-vecinos [7] (Sección II.C), permitiendo observar sugerencias personalizadas para cada cliente.

\section{Estado del Arte}

\section{A. Actualidad de NFC en el mundo comercial}

Los últimos datos de la consultora Juniper Research, presentados el 13 de abril de 2016, exponen buenos tiempos para el comercio electrónico. El estudio realizado para un periodo comprendido entre los años 2016-2020, revelan que sectores como la banca digital y la industria en general tendrán un importante crecimiento gracias a eventos especiales como el Cyber Monday o el día del soltero.

Por otra parte, el informe expone que este crecimiento impulsará el mercado de pagos por NFC, donde los líderes indiscutibles serán Samsung y Apple, fomentando el uso de terminales de pago electrónico sin contacto y ofreciendo una gran cantidad de servicios, adaptándolos a las necesidades de consumo [8].

Así como Samsung y Apple, existen gran variedad de compañías en la actualidad que le apuestan a tecnologías como NFC, ya que ofrecen una extensa lista de variables que permiten mejorar procesos y reducir tiempos de espera. Adicionalmente, es una herramienta que proporciona la posibilidad de gestionar inventarios y generar tendencias de compra, aportando datos de gran utilidad para la toma de decisiones empresariales.

\section{B. $N F C$}

NFC o comunicación de campo cercano (Near Field Communication) es una tecnología desarrollada por SONY y NXP (Formerly Philips Seminconductors) mundialmente reconocida y, desde diciembre 2003, se encuentra acreditada bajo el estándar ISO/IEC 18092, que define los modos de comunicación para la interfaz y el protocolo de comunicación (NFC IP-1) que trabajan bajo una frecuencia central de 13,56MHz (estándares NFC Fig. 1) [9].

La comunicación ocurre entre dos dispositivos compatibles con una frecuencia de $13.56 \mathrm{MHz}$, el protocolo, por su parte, distingue entre dos modos de operación, los cuales son modo activo y modo pasivo. En el modo activo de la comunicación, los dos dispositivos usan su propia energía para generar su propio campo para transmitir los datos [11]. En el modo pasivo de la comunicación, sólo uno de los dispositivos genera el campo, mientras el otro usa la energía creada por el dispositivo activo (Tabla 1). 


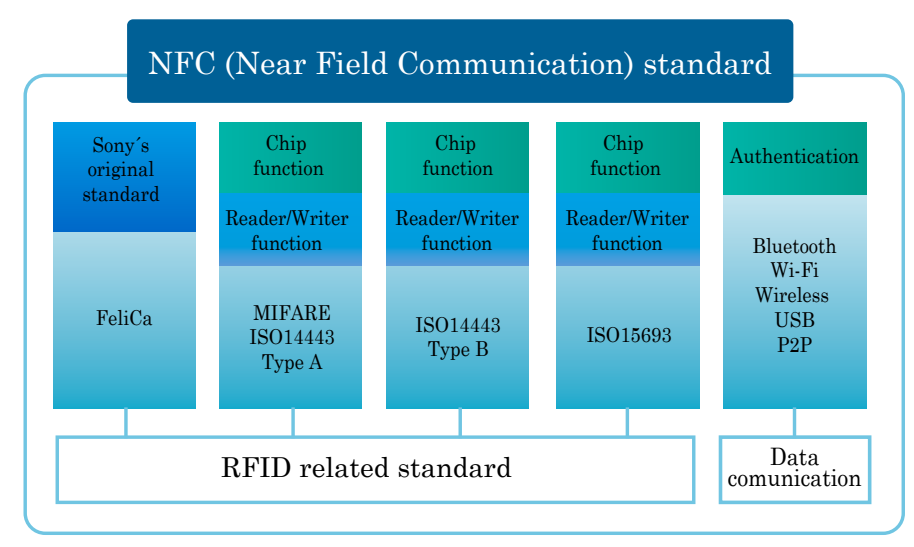

Fig. 1. Estandares bajo los que rige NFC.

Fuente: [10].

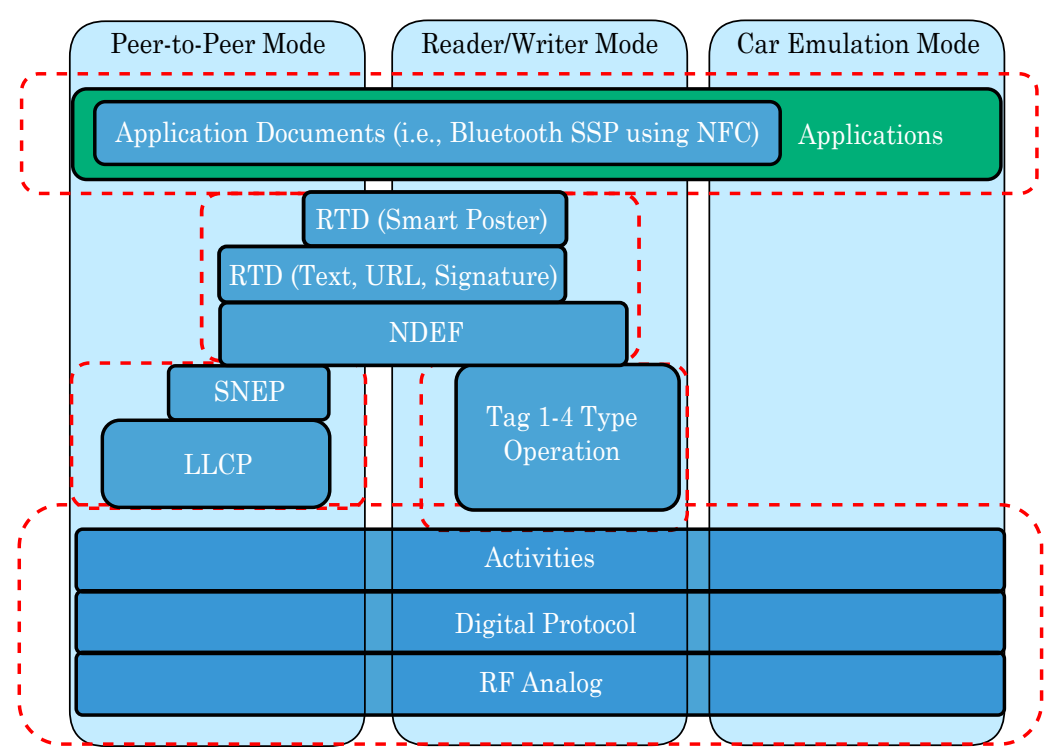

Fig. 2. Especificación de arquitectura modo de operación NFC.

Fuente: [14].

Tabla 1. Modo de Comunicación (aCtivo vs pasivo).

\begin{tabular}{|c|c|c|c|}
\hline Dispositivo A & Dispositivo B & RF Generación de Campos & Modo de Comunicación \\
\hline Activo & Activo & Generado por ambos dispositivos & Modo activo \\
\hline Activo & Pasivo & Generado sólo por el dispositivo A & Modo pasivo \\
\hline Pasivo & Activo & Generado sólo por el dispositivo B & Modo pasivo \\
\hline
\end{tabular}

Fuente: Autores.

Existen tres tipos de dispositivos NFC que pueden estar inmersos en la comunicación: móviles, etiquetas y lectores. El primero corresponde a los diferentes dispositivos que llevan incorporada la tecnología, como teléfonos y tabletas; la etiqueta NFC, por su parte, es un elemento pasivo complementario que contiene información relevante y puede ser utilizada para guardar datos que permitan el acceso a canales según sea el ambiente en el que se encuentre; y, por último, el lector NFC, el cual es común encontrarlo en tiendas o centros con terminales de pago. 
La tecnología NFC opera en tres diferentes estados de funcionamiento, los cuales son la lectura/escritura, peer-to-peer y card emulation (especificación arquitectura modo de operación Fig.2) [12]. Cada modo utiliza diferentes interfaces de comunicación (ISO/IEC 14443, NFCIP-2), así como diferentes requisitos técnicos que permiten su operación [13].

Modo lectura/escritura: permite a los diferentes dispositivos leer la información almacenada en las diferentes etiquetas proporcionadas por entidades comerciales, ya sea publicidad o consulta de información de un producto específico (compatible con NFC-A, NFC-B y NFC-F).

Modo peer-to-peer: Permite a dos dispositivos habilitados para NFC comunicarse entre sí para intercambiar información y compartir diferentes tipos de archivos.

Modo de emulación de tarjeta: permite la comunicación de forma bidireccional entre dos dispositivos, como tarjetas inteligentes, permitiendo a los usuarios realizar transacciones con un sólo toque.

\section{Sistemas de recomendación}

Con el paso de los años, son los sistemas de recomendación los que han venido adquiriendo mayor importancia en el mundo del comercio electrónico. Tienen como objetivo ayudar a los usuarios a elegir los productos más adecuados para sus gustos y necesidades, bien sea, ofreciendo una valoración de la utilidad de determinado producto o bien recomendando directamente un conjunto de ellos adecuados a sus preferencias.

En la actualidad, los sistemas de recomendación gozan de fama gracias a su capacidad para ofrecer recomendaciones personalizadas, ya que mantienen un perfil independiente para cada usuario [15].

\section{1) Tipos de sistemas de recomendación}

Los sistemas de recomendación dependen de una gran cantidad de variables que permiten organizar la información y presentarla de acuerdo con tendencias, gustos o, incluso, dependiendo de la región del usuario [16]; así es que, a través de los años, los sistemas de recomendación se han venido clasificando en:

- Sistema de recomendación con filtrado basado en contenido.

En este tipo de sistemas las recomendaciones se basan en un valor que es asignado por los diferentes usuarios (Ejemplo: YouTube).

\section{- Sistema de recomendación con filtrado} demográfico.

En este tipo de sistemas, las recomendaciones se basan en características propias del usuario como: sexo, edad, ubicación geográfica, etc.

\section{- Sistema de recomendación con métodos de filtrado híbrido.}

En este tipo de sistemas, las recomendaciones se asignan a través de la unión de los diferentes tipos de sistemas de recomendación mencionados con anterioridad, aumentando su eficacia y siendo más exactos con los datos suministrados al cliente.

\section{- Sistema de recomendación basado en filtrado colaborativo.}

En este tipo de sistema, las recomendaciones se basan en encontrar usuarios que contengan gustos similares con el objetivo de ofrecer productos que ellos hubieran elegido previamente.

\section{- Sistemas de recomendación basados en filtrado colaborativo (K-vecinos).}

Los sistemas de recomendación basados en filtrado colaborativo se clasifican en: los basados en memoria y los basados en modelos, que permiten realizar sugerencias a partir de diferentes técnicas (Tabla 2).

TABla 2. TÉCniCAS EMPleadas EN LOS SISTEMAS DE RECOMENDACIÓN.

\begin{tabular}{|c|c|}
\hline Basados en Memoria & Basados en Modelos \\
\hline K-Vecinos & Algoritmos genéticos \\
\hline JMSD & Redes neuronales \\
\hline MSD & Lógica difusa \\
\hline SVD & SVD \\
\hline Correlación & \\
\hline Coseno & \\
\hline
\end{tabular}

Fuente: Autores

Por su parte, los métodos basados en memoria emplean medidas de similitud para determinar el parecido entre una pareja de usuarios, mientras que los métodos basados en modelos generan un patrón de comportamiento a partir de votaciones para crear un modelo a través del cual se establece un conjunto de usuarios similares al usuario activo.

\section{Técnica k-vecinos}

La técnica de los k-vecinos es considerada como un método de clasificación fácil de entender y fácil de implementar [17] que se encuentra en el top 10 de los algoritmos de minería de datos [18]. En 1951, Fix y Hodges introdujeron un método no paramétrico para la clasificación de patrones, el cual se conoce como k-vecinos. Su esencia se centra en buscar usuarios con tendencias y características semejantes que permitan obtener información relevante para recomendar productos a un usuario activo; ya sean películas, música o productos de adquisición por sitios web o centros comerciales. 
Su éxito depende de una serie de variables que deben ser recolectadas con anticipación. En un primer instante, el usuario debe aportar información con la que el sistema realizará una serie de cálculos que permitan obtener aquellos usuarios (vecinos) con una tendencia muy parecida. Para obtener la información, el sistema pone a disposición del usuario un esquema que permite cuantificar el nivel de satisfacción de un producto, generalmente las diferentes plataformas asignan un rango de 1-5, teniendo (1) como nivel de satisfacción muy bajo y (5) como muy alto [19].

Una vez se obtienen los datos suministrados por los diferentes usuarios, estos se listan en una matriz de votos con la que se procede a calcular la similitud entre usuarios. El cálculo obtenido puede llegar ubicarse en un rango de entre -1 a 1 , indicando directamente el grado de similitud; para obtener el cálculo se pueden utilizar diferentes tipos de medidas, entre las más conocidas se encuentran la Correlación de Pearson y la del Coseno [20].

\section{E. Algoritmo de K-vecinos}

La implementación de la técnica de los k-vecinos en un algoritmo produce cuatro pasos que permiten establecer el grado de similitud entre varios usuarios (Fig. 3) [21]:

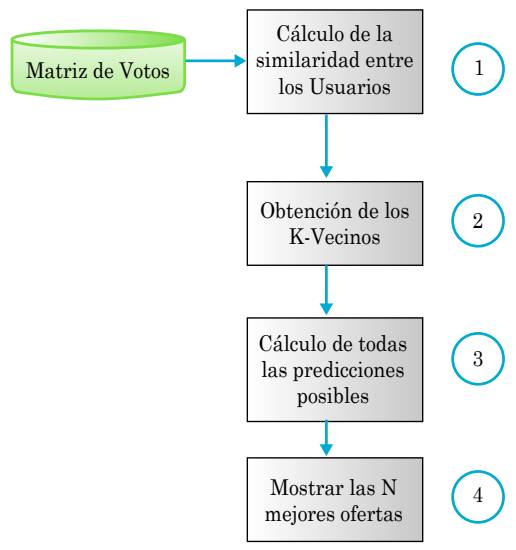

Fig. 3. Flujo de trabajo del algoritmo de los k-vecinos. Fuente: Autores.

Cálculo de similitud entre usuarios. De acuerdo con una serie de técnicas, se procede a calcular la similitud entre diferentes usuarios.

Calcular los k-vecinos. A partir de los datos obtenidos con las técnicas se obtienen los usuarios con mayor grado de similitud.

Calcular las predicciones de los ítems. Teniendo en cuenta los usuarios se procede a predecir el valor que el usuario activo podría ofrecer sobre artículos que aún no ha valorado.

Realizar las recomendaciones. Tras realizar los diferentes cálculos se procede a seleccionar los $\mathrm{N}$ artículos con mayor puntaje que pueden ser considerados para el usuario activo.

\section{III.Introducción al Diseño del Sistema}

El presente proyecto se realiza con una base de datos alimentada a partir de productos que normalmente se encuentran en un centro comercial o almacén de cadena, adicionalmente, se trabajan con una muestra de cincuenta usuarios con tendencias de compra diferentes, así como ubicación, creencias y estrato entre otras variables que permiten la ejecución del proyecto en un ambiente que no es controlado, permitiendo así observar la interacción del algoritmo con los usuarios.

\section{A. Arquitectura del sistema}

La arquitectura del sistema está diseñada para que los dispositivos móviles o clientes puedan acceder a los servicios que se prestan remotamente a través de un servidor y una base de datos (Fig. 4).

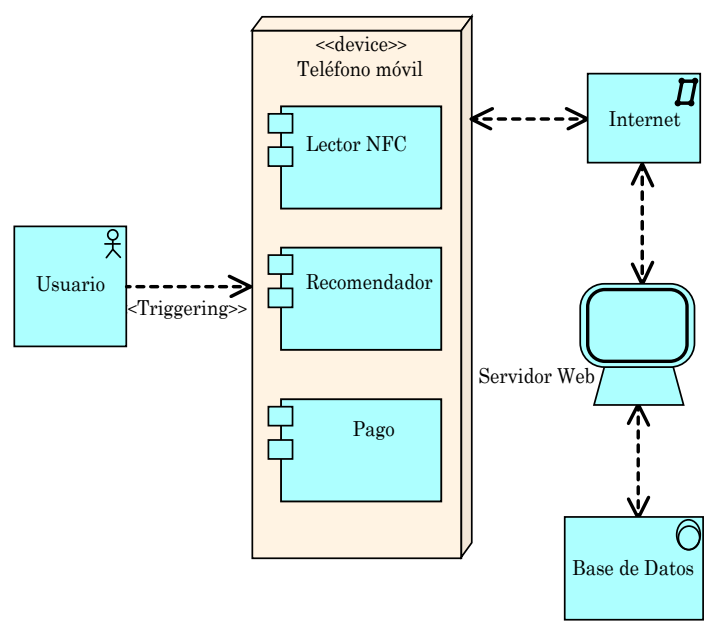

Fig. 4. Arquitectura del sistema. Fuente: Autores.

En esta arquitectura, cada cliente conoce el servidor con el cual se va a comunicar y sabe qué servicios presta el mismo, pero el servidor no necesariamente conoce a cada cliente que se conecte a él y tampoco puede determinar qué servicios prestarle a cada uno de ellos, esta decisión se toma de acuerdo con la dinámica de cada usuario y las acciones de éste en el sistema.

El principal actor en el sistema es el usuario, el cual es el encargado de desencadenar los eventos al interactuar con la aplicación en su teléfono móvil, así mismo, el usuario es el eslabón final en la cadena de eventos, puesto que recibe una retroalimentación del sistema de acuerdo con su actividad y preferencias.

La aplicación es el medio por el cual un usuario accede a los servicios prestados por el sistema. Ésta tiene tres componentes principales que se encargan de la lectura de las etiquetas NFC, de los pagos electrónicos y de establecer las recomendaciones de productos de acuerdo con el perfil del usuario. 
La entrada de información al sistema ocurre cuando se escanea una producto al realizar una compra o calificar los productos comprados; esta información se enviará al servidor y, finalmente, a la base de datos para constituirse como el recurso principal para que el algoritmo de recomendación realice los cálculos correspondientes y pueda generar una serie de productos sugeridos de acuerdo con el perfil único de cada cliente.

La aplicación se comunica a través de internet con un servidor, el cual se encarga de identificar si la información enviada corresponde a una lectura, una compra o una calificación de un producto. Una vez identificados estos parámetros, el servidor genera dinámicamente las consultas para enviarlas a la base de datos y, de esta forma, moldear el sistema de información que alimentará al sistema de recomendación.

Adicionalmente, el servidor recibe el resultado de los cálculos del algoritmo para generar una consulta a la base de datos. El resultado de ésta debe ser mapeado por el servidor y devuelto a la aplicación como una lista de productos sugeridos para ser presentados al usuario.

La base de datos es el lugar donde se almacenan los datos de los usuarios y los productos. La información almacenada en ella por sí sola no tiene mayor significado, por esta razón, es necesario implementar un servidor que actúe como interme- diario entre la aplicación móvil y la base de datos para poder conseguir únicamente la información que resulte relevante y que sea útil para la toma de decisiones por parte del usuario y que represente un valor agregado para todo el sistema.

En la base de datos no se almacena información comercial sobre inventarios, contabilidad, transacciones, etc. La información almacenada es únicamente para soportar el sistema de información para el algoritmo de recomendación, información sobre productos, cantidad de consultas, compras y promedio de calificaciones por producto.

Se decidió implementar el algoritmo en cada cliente y no en el servidor para evitar una carga excesiva en éste y con el fin de disminuir los requerimientos en cuanto a capacidad de almacenamiento de la base de datos, puesto que sería necesario guardar el perfil de cada cliente y relacionarlo con la información de los productos.

\section{B. Diagrama de clases}

La aplicación móvil se desarrolló para Android [22], implementando los servicios para la comunicación por medio de http (Hyper Text Tranfer Protocol) [23], la lectura por medio de $N F C$ y los medios de pago electrónicos. En la fig. 5 se puede apreciar el diagrama de clases de la aplicación.
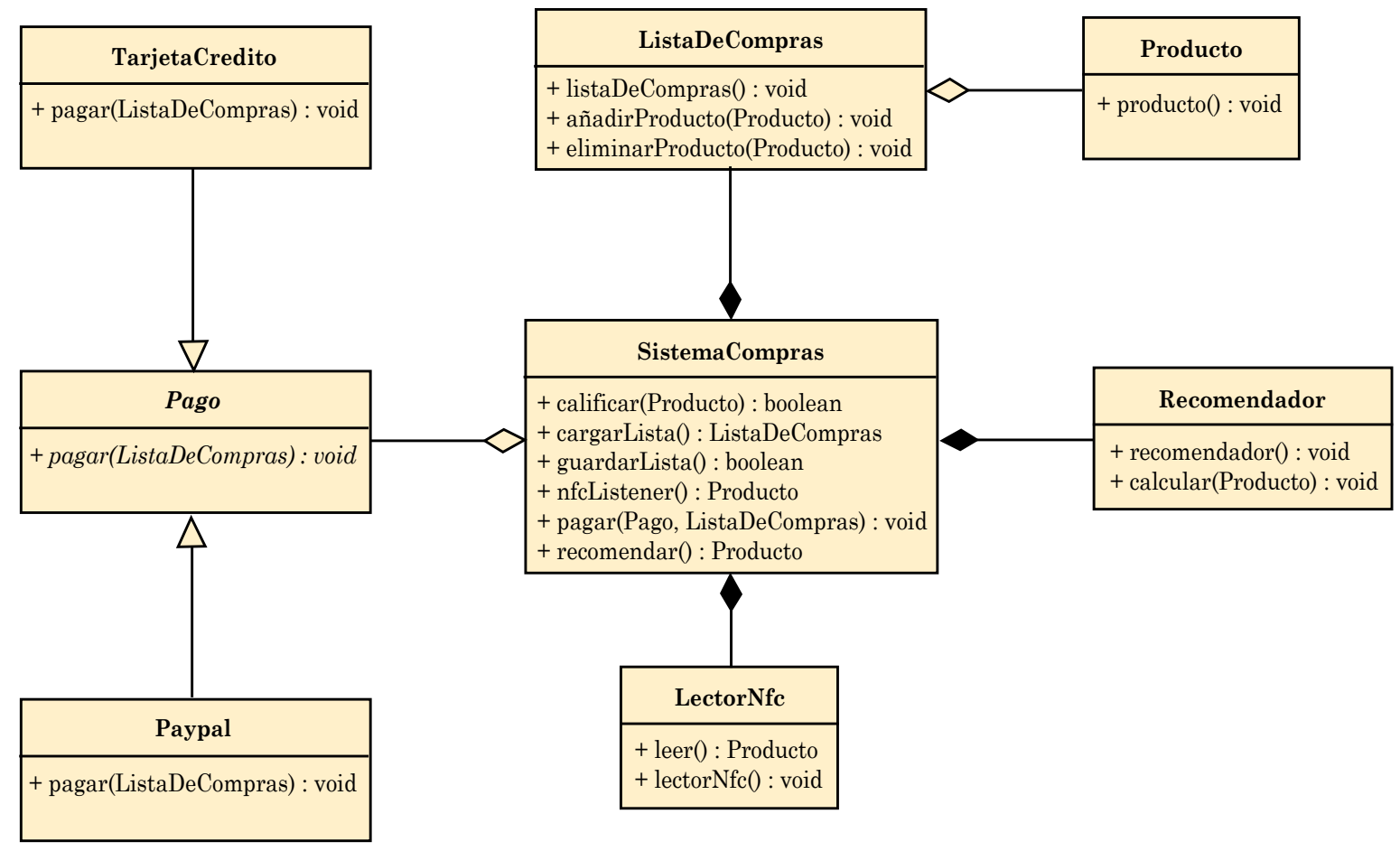

Fig. 5. Diagrama de clases. Fuente: Autores. 
La clase principal, llamada SistemaCompras, implementa una lista de compras por medio de una relación de composición con la clase ListaDeCompras, y ésta a su vez, con la clase Producto. Esto se debe a que la aplicación, desde su inicio, crea por defecto una lista de compras vacía, la cual se va llenando con productos a medida que el usuario escanea etiquetas.

La aplicación por defecto crea el Lector NFC y una relación con el sistema de recomendación, el cual se queda a la espera de recibir actualizaciones en la actividad de los usuarios para realizar los cálculos de las recomendaciones de acuerdo con el perfil de cada cliente.

Finalmente, se establece una relación de agregación con la clase abstracta Pago; esta clase define una estructura general para los diferentes medios de pago implementados y, a su vez, permite la implementación futura de nuevos medios. Las clases Paypal y Tarjeta de crédito son una implementación concreta de un medio de pago por medio de la herencia de la clase abstracta Pago.

\section{MSD, mínimos cuadrados}

$\operatorname{sim}(x, y)=1-\left(\frac{1}{\# B x y}\right) \sum_{i E I u}\left(\frac{r x y-r y i}{\max -\min }\right)^{2}[0.1]$

Siendo \#Bxy el número de productos que ambos usuarios han votado (y que tiene que ser necesariamente mayor que 0); siendo rxy y ryi los votos emitidos por los usuarios $x$ e $y$, respectivamente, y siendo $\max$ y $\boldsymbol{m i n}$ las notas máximas y mínimas que los usuarios han emitido.

\section{Coseno}

Partiendo de la calificación que una pareja de usuarios realizan a un mismo producto, se genera un vector donde el componente $X$ es el usuario $A$ y el componente en $Y$ es el usuario $B$; el coseno del ángulo indicará el grado de similitud entre los usuarios. Esta medida es apta para sistemas de recomendación basados en productos.

$$
=\frac{\operatorname{sim}(x, y)}{\sqrt{\sum_{i E B x y} r^{2} x y * \sqrt{\sum_{i E B x y} r^{2} y i}}}[0,1]
$$

\section{E. Correlación de Pearson}

$$
=\frac{\operatorname{sim}(x, y)}{\sqrt{\sum_{i E x y}(r x y-r x)^{\wedge} 2} \cdot \sum_{i E B x y}(r y i-r y) 2}
$$

Adicional a los parámetros anteriores, $\boldsymbol{r} \boldsymbol{x}$ y $\boldsymbol{r} \boldsymbol{y}$ son los promedios de votos del usuario $\boldsymbol{x}$ e $\boldsymbol{y}$, respectivamente.

Para la definición de la similitud con el usuario activo es necesario definir una función de similitud para encontrar los usuarios que tienen características similares. Para el respectivo proyecto se trabaja con el método de correlación de Pearson (basado en votos), mencionado con anterioridad, al ser uno de los que mejor se adapta al modelo propuesto y presenta mejores resultados en proyectos trabajados en ambientes web y comercio electrónico debido a que la correlación de Pearson emplea un promedio de votos que le permite tratar los casos en los que un usuario tiende a otorgar puntuaciones iguales en la mayoría de los productos, o también los casos en los que no se realizan calificaciones, a diferencia de los mínimos cuadrados [24].

Los valores posibles de similitud varían desde -1 (correlación negativa) a 1 (correlación positiva) donde:

$\operatorname{Si} \operatorname{Sim}(a, b)=1$, se considera una correlación positiva perfecta.

Si $0<\operatorname{Sim}(a, b)<1$, se considera como una correlación positiva donde el grado de similitud es mayor conforme se próxima a 1 .

$\operatorname{Si} \operatorname{Sim}(a, b)=0$, se considera que no existe una correlación entre a y b.

$\mathrm{Si}-1<\operatorname{Sim}(a, b)<0$, se considera que existe una correlación negativa.

Si $\operatorname{Sim}(a, b)=-1$, se considera que existe una correlación negativa perfecta, lo que indica un comportamiento inversamente proporcional entre las variables [25,26].

Para el modelo propuesto se trabaja inicialmente con una base de datos que involucran el nombre del producto, el precio, las visitas, las compras, las calificación promedio, entre otras.

Siguiendo con el procedimiento mencionado en la sección III, se debe establecer una matriz de votos que relaciona los productos con los usuarios y sus respectivos niveles de satisfacción. Para el caso en estudio, el sistema seleccionó 5 usuarios, teniendo en cuenta que el tamaño de la muestra se encontraba acotado por las características demográficas del usuario activo (Tabla 3).

Adicionalmente, se filtró la cantidad de productos a evaluar basándose en el número de compras y consultas que presentaban, de esta forma se logra introducir al sistema tres diferentes parámetros para mejorar la exactitud del algoritmo.

Se puede observar que el valor más alto es el de la correlación entre Carlos y María; igualmente, se puede observar que aparecen valores de correlación negativos, los cuales establecen que existe una correlación inversamente proporcional entre los valores comparados de sus respectivos usuarios (Tabla $3)$. 
Tabla 3. Datos insertados en la base de datos

\begin{tabular}{|c|c|c|c|c|c|}
\hline $\begin{array}{c}\text { Usuario/ } \\
\text { Producto }\end{array}$ & $\begin{array}{c}\text { Cable } \\
\text { HDMI }\end{array}$ & Nokia & $\begin{array}{c}\text { MacBook } \\
\text { Air }\end{array}$ & $\begin{array}{c}\text { B. } \\
\text { ray }\end{array}$ & $\begin{array}{c}\text { H. } \\
\text { Thea }\end{array}$ \\
\hline Carlos & 5 & 3 & 4 & 4 & - \\
\hline María & 3 & 1 & 2 & 3 & 3 \\
\hline Andrés & 4 & 3 & 4 & 3 & 5 \\
\hline Juan & 3 & 3 & 1 & 5 & 4 \\
\hline Pablo & 1 & 5 & 5 & 2 & 1 \\
\hline
\end{tabular}

Fuente: Autores

La Fig. 6, por su parte, muestra la relación de la puntuación de los diferentes usuarios con los productos que han sido puntuados. En la tabla 4 se presentan los todos los valores posibles de correlación.

TABla 4. VALORES DE CORRELACióN

\begin{tabular}{|c|c|c|c|c|c|}
\hline & Carlos & María & Andrés & Juan & Pablo \\
\hline Carlos & $\mathrm{x}$ & 0,85 & 0,71 & 0,00 & $-0,79$ \\
\hline María & 0,85 & $\mathrm{x}$ & 0,47 & 0,49 & $-0,90$ \\
\hline Andrés & 0,71 & 0,47 & $\mathrm{x}$ & $-0,16$ & $-0,47$ \\
\hline Juan & 0,00 & 0,49 & $-0,16$ & $\mathrm{x}$ & $-0,64$ \\
\hline Pablo & $-0,79$ & $-0,90$ & $-0,47$ & $-0,64$ & $\mathrm{x}$ \\
\hline
\end{tabular}

Fuente: Autores.

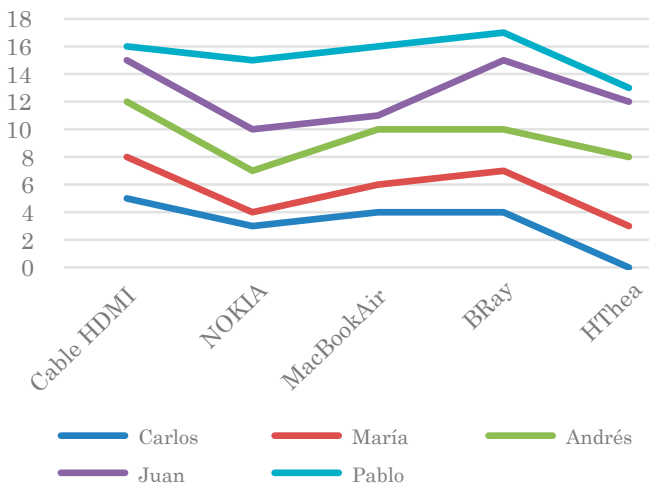

Fig. 6. Gráfico de valoraciones a partir de un conjunto de datos de prueba. Fuente: Autores

Una vez que se ha encontrado que María es el usuario con mayor similitud se puede decir que es uno de los k-vecinos de Carlos, entonces se procede a calcular la predicción mediante la técnica de los k-vecinos mencionada con anterioridad (sección III), para lo que se aplica la siguiente ecuación [27]-[29]:

$\operatorname{pred}(a, b)=V a+\frac{\sum \operatorname{sim}(a, b) *(V b p-V b)}{\sum \operatorname{sim}(a, b)}$
La predicción obtenida para el producto 5 es de 4.8 según la ecuación (4), lo que indica que el usuario activo tiene una probabilidad cercana al $96 \%$ de estar interesado en dicho producto.

Lo que significa que entre mayor sea el grado similitud entre los usuarios, la predicción de la valoración del usuario A sobre el producto será más precisa [30].

A partir del ejemplo anterior se estableció que el algoritmo permite sugerir los diferentes productos a cada usuario, clasificando los tres productos con mayor puntaje como aquellos que aparecerán como productos recomendados en la aplicación.

\section{Funcionamiento de la Aplicación}

La aplicación se desarrolló para operar en Android 4.2 o superior. Para su funcionamiento básico sólo requiere que el dispositivo tenga soporte para NFC, de esta forma se podrá hacer uso de las funciones relacionadas con la creación de listas de compra. Para poder hacer uso de la función principal del sistema de recomendación es absolutamente necesario contar con una conexión a internet al momento de utilizar la aplicación.

Una vez que se inicia la aplicación, el usuario se encuentra con la ventana principal, la cual le presenta un campo en el que se muestra el valor total a pagar por los artículos de la lista de compras, también se presentan unos botones que le permiten tomar decisiones, como pagar, guardar y borrar listas de compra. Finalmente, se presenta un espacio en blanco que corresponde al espacio reservado para mostrar los artículos añadidos a la lista de compras, que por defecto se encuentra vacía (Fig. 7).

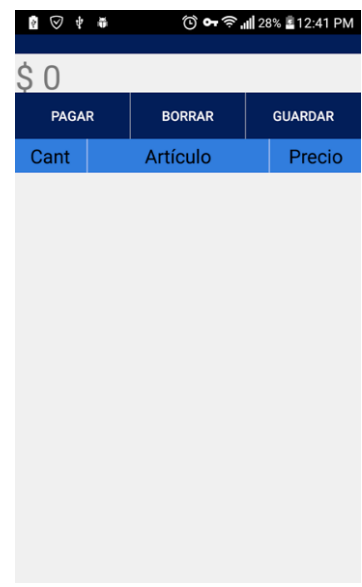

Fig. 7. Pantalla inicial Fuente: Autores

Una vez que el usuario empieza a escanear productos, estos aparecen en la lista de compras (Fig. 8). 


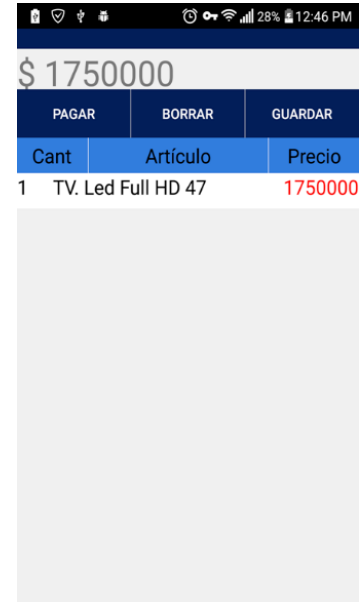

Fig. 8. Añadiendo productos. Fuente: Autores

A medida que los usuarios escanean productos la aplicación se comunica con el servidor para enviarle las actualizaciones de cada nuevo producto que se añade a la lista de compras. Esta información es de vital importancia puesto que es uno de los parámetros que usa el algoritmo para realizar los cálculos para ofrecerle al usuario recomendaciones personalizadas.

Otro de los parámetros necesarios para los cálculos del algoritmo es la calificación que le otorgan los usuarios a un producto que han comprado. Cuando el usuario termina de crear su lista de compras y decide pagar, se despliega una nueva ventana en la cual se le presentan los productos que compró y una barra de calificaciones para que evalúe cada artículo en una escala de uno a cinco (Fig. 9).

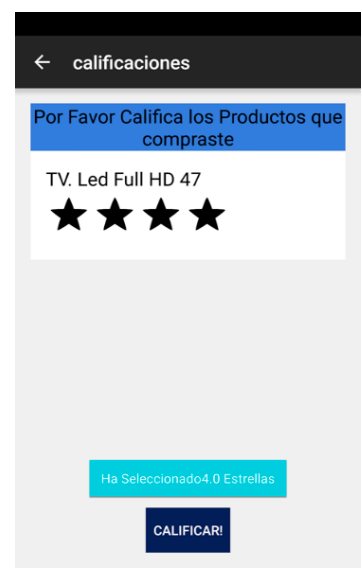

Fig. 9. Barra de calificaciones. Fuente: Autores

De forma simultánea al procesamiento de las calificaciones, la aplicación envía los productos comprados y la cantidad de cada uno de ellos, para constituir el último de los parámetros que alimentan al sistema de información.
En cualquier momento, el usuario puede consultar cuáles son los productos que el sistema le sugiere. Cada evento que desencadena el usuario provoca un nuevo cálculo del algoritmo y una nueva actualización en las sugerencias que el sistema realiza (Fig. 10).

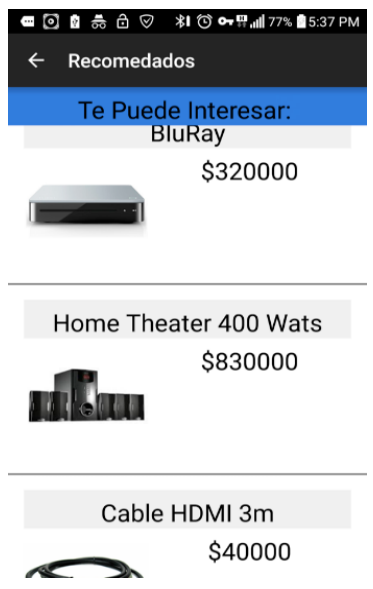

Fig. 10. Productos recomendados. Fuente: Autores

\section{Trabajos Futuros}

Para la continuación de este proyecto se tiene planeado lanzar una versión beta de la aplicación para dar inicio a la fase de pruebas con usuarios reales y estudiar el desempeño de los servicios prestados en un ambiente menos controlado. Igualmente, se espera recibir la retroalimentación de los usuarios para realizar los ajustes que sean necesarios al sistema. Se tiene proyectado que durante el segundo semestre de 2016 se lleve a cabo la etapa de pruebas, despliegue y mantenimiento para poder contar con una versión productiva.

\section{Conclusiones}

La implementación de la técnica de los k-vecinos en un algoritmo de recomendación permite suministrarle al cliente una serie de recomendaciones con un nivel de seguridad mucho más alto que el que pueden proveer otros sistemas similares, puesto que este algoritmo realiza cálculos teniendo en cuenta múltiples parámetros y contrasta los resultados obtenidos para otros usuarios logrando encontrar los artículos con un mayor grado de similitud con el perfil del cliente.

El algoritmo permite realizar una selección más precisa de las recomendaciones partiendo de una gran cantidad de productos, como se muestra en la Fig. 10. El algoritmo parte de una muestra de productos similares, complementarios y otros no relacionados, pero luego de aplicar las fórmulas se obtiene que la recomendación se realiza únicamente con los productos complementarios que obtuvieron mayor calificación, como se muestra en la Fig.10. 
Esto marca una notable diferencia con la mayoría de sistemas de recomendación del mercado que sólo se limitan a sugerir los productos más vendidos, mejor calificados o de la misma categoría.

La implementación de sistemas complementarios y tecnologías innovadoras a las plataformas de comercio electrónico les proporcionan a los comerciantes una ventaja estratégica frente a sus competidores puesto que les permiten posicionar una mayor cantidad de productos al alcance de sus clientes y aprovechar el auge de las tecnologías móviles para aumentar sus ganancias.

La técnica de correlación de Pearson resulta ser la más adecuada para ser implementada en sistemas informáticos, en especial en ambientes web y móviles debido a que facilita el cálculo a partir de los usuarios con características similares de compra, a diferencia de las técnicas del coseno y mínimos cuadrados que se aplican a ambientes donde el producto es la esencia del cálculo.

Al diseñar desde su arquitectura la aplicación para que el sistema de recomendación opere de forma dinámica, se le proporciona al sistema la capacidad de presentar recomendaciones en tiempo real y aprovechar el pequeño intervalo de tiempo en el que el cliente es receptivo a las sugerencias y muestra interés en adquirir nuevos productos como se sugiere en el artículo [3] (sección I).

\section{Agradecimientos}

Esta investigación se lleva a cabo en el marco de las líneas de investigación del Grupo de Investigación GIIRA, Facultad de Ingeniería, de la Universidad Distrital Francisco José de Caldas.

\section{Referencias}

[1] R. Gutierrez, "La era digital revoluciona el mundo del Shopper", Marketing News, E.59, pp. 20-21,2015.

[2] E.E. Izaguirre. Sistemas de recomendación en Apache Spark. Academia e., 2015. [Online]. Disponible en: http:// academica-e.unavarra.es/handle/2454/19015

[3] C.A Gomez y N. Hunt, "The Netflix Recommender System: Algorithms, Business Value, and Innovation”. ACM Transactions on Management Information Systems, vol. 6, no. 4 pp. 13,3-13,5, Feb. 2016. https://doi.org/10.1145/2843948

[4] D. Hongwei, "NFC Technology: Today and Tomorrow". International Journal of Future Computer and Communication, vol. 2 , no. 4 pp.351-353, 2013. https://doi.org/10.7763/ IJFCC.2013.V2.183

[5] ISO. International Standard ISO/IEC 18092:2013 Technica Corrigendum 1, 2004. [Online]. Disponible en: http://www. iso.org/iso/catalogue_detail.htm?csnumber $=38578$

[6] V. Nikitin y S. Lazar, "An Overview of Near Field UHF RFID". IEEE International Conference on RFID, Texas, USA, pp. 6-10, Mar. 2007. https://doi.org/10.1109/rfid.2007.346165

[7] J. Maillo y I. Triguero, "Un enfoque MapReduce del algoritmo k-vecinos más cercanos para Big Data”. ACM, vol. 7, pp. 971-980, 2003

[8] S. Smith, Digital Commerce Transactions to surge: Market to see Substantial Contributions from e-commerce Purchases, 2016. [Online]. Disponible en: http://www.juniperresearch. $\mathrm{com} / \mathrm{press} /$ press-releases/digital-commerce-transactions-tosurge-reaching-ov
[9] NFC-WORLD, Near Field Communications (NFC), 2017. [Online]. Disponible en: http://www.nfc-world.com/en/ about/01.html

[10] NFC-WORLD, Near Field Communications (NFC), 2017. [Online]. Disponible en: http://www.nfc-world.com/en/ about/01.html

[11] V. Coskum y B. Ozdenizci, (2013). "A Survey on Near Field Communication (NFC) Technology". Wireless Personal Communications, 71, pp. 2259-2294, 2013. https://doi.org/10.1007/ s11277-012-0935-5

[12] NFC FORUM, NFC Devices Operate in 3 Modes, 2017. [Online]. Disponible en: http://nfc-forum.org/what-is-nfc/what-itdoes/.

[13] F.V. López, "Técnicas eficientes para la recomendación de productos basadas en filtrado colaborativo" (Tesis de Doctorado). Departamento de tecnologías de la información y de las comunicaciones, Universidad de Coruña, España, 2013.

[14] NFC FORUM, NFC Forum Specification Architecture, 2017 [Online]. Disponible en: http://nfc-forum.org/our-work/specifications-and-application-documents/specifications/.

[15] J.J. Fuentes, "Sistema Recomendador Turístico" (Máster en Ingeniería Telemática). Departamentos de Redes e Ingeniería, Universidad de Vigo, Orense, España, 2009.

[16] D.G. Sánchez y I.E. Piza. "Un algoritmo de clasificación in cremental basado en los k-vecinos más similares para datos mezclados", Revista-Facultad de Ingeniería Universidad de Antioquia, vol. 67, pp. 19-25, 2013.

[17] X. Wu, V. Kumar y J. Ross Quinlan. "Top 10 algorithms in data mining", Knowledge and Information Systems, vol. 14, un. 14:1, December 2007. https://doi.org/10.1007/s10115-0070114-2

[18] S. Thirumuruganathan, A Detailed Introduction to K-Nearest Neighbor, 2010. [Online]. Disponible en: https://saravananthirumuruganathan.wordpress.com/2010/05/17/a-detailed-introduction-to-k-nearest-neighbor-knn-algorithm/.

[19] Bobadilla, J. (2010). "A new collaborative filtering metric that improves the behavior of recommender systems", KnowledgeBased Systems, vol. 23, pp. 520-528, July 2010. https://doi. org/10.1016/j.knosys.2010.03.009

[20] F.S. Pita y D.S. Pertega, Relación entre variables cuantitativas, 2001. [Online]. Disponible en: https://www.fisterra.com/ mbe/investiga/var_cuantitativas/var_cuantitativas2.pdf.

[21] J.T. Ekstrand y J.A. Konstan "Collaborative Filtering Recommender Systems", Now Foundations and Trends, vol. 23, pp. 291-320, 2011. https://doi.org/10.1561/1100000009

[22] M. Spreitzenbarth y F. "Having a deeper look into android applications". Presentado en: Proceedings of the 28th Annual ACM Symposium on Applied Computing, New York, USA 2013, pp.16-18. https://doi.org/10.1145/2480362.2480701

[23] R. Fielding y J. Gettys, Hypertext Transfer Protocol HTTP/1.1., 1999. [Online]. Disponible en: https://tools.ietf. $\mathrm{org} / \mathrm{html} / \mathrm{rfc} 2616$

[24] G.E. Ainhoa, "Uso del contexto social en estrategias de marketing para sistemas recomendadores" (Máster en Investigación). Facultad de Informática, Universidad Complutense, Madrid, España, 2013.

[25] Sarwar y G. Karypis. "Collaborative Filtering Recommen dation Algorithm", Advanced Science and Technology Letters, vol. 111, pp. 143-146, 2015. https://doi.org/10.14257/ astl.2015.111.27

[26] N.K Hayder y A.L. Behadili. "Classification Algorithms for Determining Handwritten Digit", Electrical and Electronic Engineering, vol. 12, pp 96-99, 2010

[27] O.J. Carratala y E.V. Ruiz. "Algoritmos de búsqueda de vecinos más próximos en espacios métricos" (Tesis Doctoral), Departamento de Sistemas Informáticos y Computación, Universidad Politécnica de Valencia, España, pp. 123-125, 1996.

[28] J. Maillo y I. Triguero. "Un enfoque MapReduce del algoritmo k-vecinos más cercanos para Big Data”, Neurocomputing, vol. 150 , pp. 332-333, 2015

[29] J Lineberger y W. Thompson, Recommendations Engine in Layered Social Media Webpage, Patent Application Publication, pp 1-14, 2016.

[30] S.E. García y C.M. Romero, "Sistema recomendador colaborativo usando minería de datos distribuida para la mejora continua de cursos e-learning", Electrical and Electronic Engineering, vol.3, pp. 19-20, mayo 2008 . 